\title{
Optimization of Far-Field Antenna Range
}

\author{
Ondrej CERNY, Radovan DOLECEK, Petr KOPECKY, Vladimir SCHEJBAL, Vadim ZAVODNY \\ University of Pardubice, Studentska 95, 532 10, Pardubice, Czech Republic \\ vladimir.schejbal@upce.cz
}

\begin{abstract}
Measurements of test antennas are performed on antenna ranges. The operated microwave far-field outdoor range was built-up in 1970's and therefore it was not appropriate for the today measurements. Thus, it was decided to perform the complete reconstruction and testing. Some results of new ample measurement campaign are just given. The optimization of antenna range using merely measurement is very inefficient, and therefore that is done by numerical simulations. Consequently the paper surveys briefly electromagnetic wave propagation over irregular terrain. The physical optics approximation of vector problem was chosen. That allows the comparison of selected numerical simulations and measurements for the reconstructed far-field range. A possibility of antenna range optimizing by using numerical simulation considering various constraints is verified.
\end{abstract}

\section{Keywords}

Microwave antenna, antenna measurements, irregular surface, physical optics, numerical analysis, experiments

\section{Introduction}

Measurements of test antennas are performed on antenna ranges [1], [2]. That are categorized as either outdoor or indoor ranges, and there are limitations associated with both of them. The illumination of the test antenna by plane wave uniform in amplitude and phase is the ideal condition for measuring far-field radiation characteristics. This ideal condition is not achievable, but it can be approximated by separating the test antenna from the illumination source by a large distance on an outdoor range. As this separation distance increases, the curvature of the spherical phasefront produced by the source antenna becomes more planar over the test antenna aperture. If the separation distance is equal to $2 D^{2} / \lambda$, where $D$ is the largest dimension of the antenna, then the maximum phase error of the incident field from an ideal plane wave is about $22.5^{\circ}$. Moreover, reflections from the ground and nearby objects are possible sources of degradation of the test antenna illumination. Therefore optimizing the planarity of the wave-fronts illu- minating the test antenna and reducing environmental reflections are the primary objectives of facilities designed to measure the far-field characteristics of antennas. As antennas increase in size or operating frequency, it becomes increasingly difficult to measure antenna properties in the far-field. Moreover, outdoor ranges are not protected from environmental conditions. That means any kind of precipitation and/or wind, which can produce undesired movements of the test or source antennas, will have a negative impact on radiation measurements.

On the other hand, determining the far-field pattern of an antenna from near-field measurements requires a mathematical transformation and correction for the characteristics of the measuring antenna [1], [3]-[7]. That could be done both by measuring of amplitude and phase or by phase-less methods, which could be much more cheaper, as only amplitudes or powers can be measured. Moreover, big problems are created for extremely large antennas considering plane-rectangular and cylindrical near-field scanners, which are well-built, very expensive and measurementtime limited. However, a great progress in the development of indoor ranges has been done.

The functional far-field outdoor range was built-up in 1970's and it is not suitable for the today measuring. Therefore, it was approved to perform the complete reconstruction and testing. To keep a reasonable paper extent, some results of new ample measurement campaign are merely given. As the optimization of antenna range using measurement is very time-consuming that could be advantageously done by numerical simulations. The paper surveys briefly electromagnetic wave propagation over irregular terrain by physical optics approximation of vector problem. To compare that with measurements, the new vertical positioner for testing of electric field vertical distribution has been designed. The comparison of numerical simulations and measurements for the reconstructed farfield range is given merely for two cases. The design of antenna far-field range for antenna measurements is rather complex task, which is analyzed [2] in detail. The aim of the paper is manifold. Firstly, a suitability of a chosen numerical approximation should be demonstrated. Secondly, a possibility of antenna range optimizing by using numerical simulation is verified considering various constraints. 


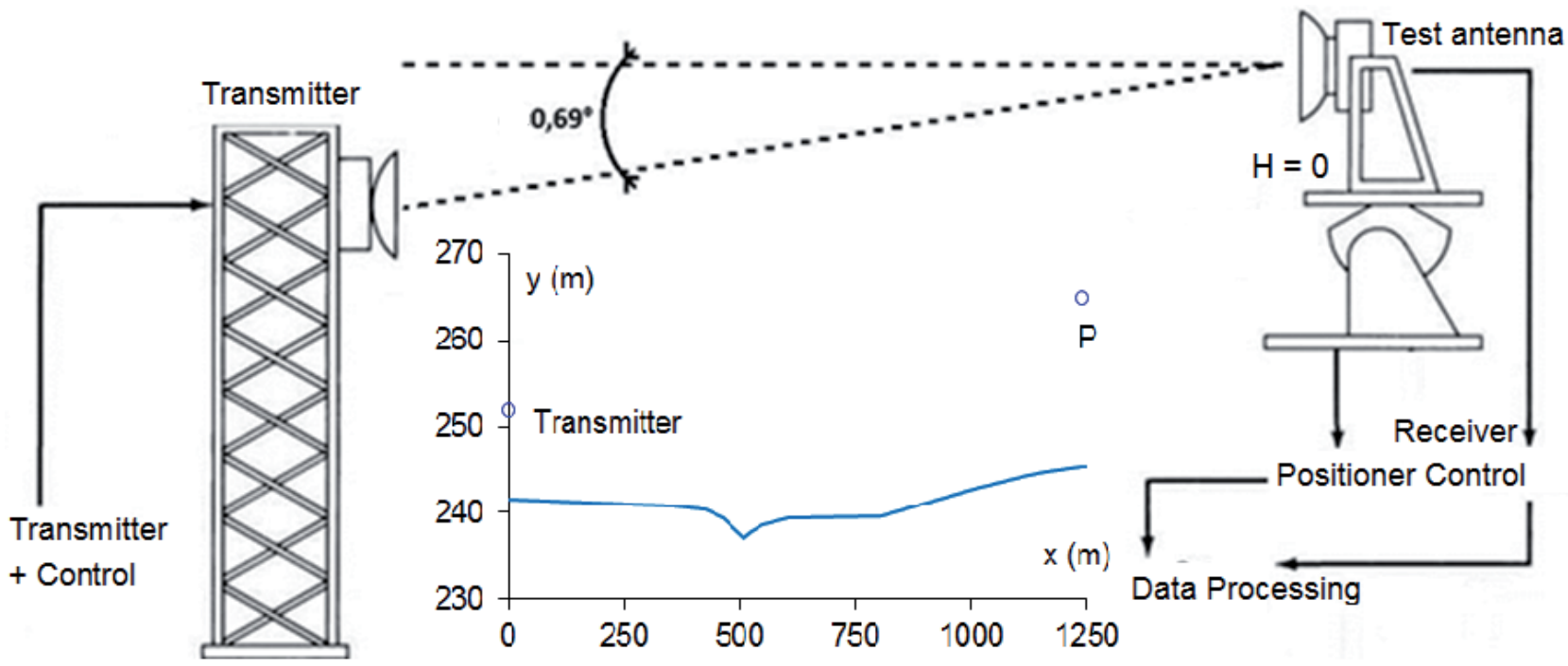

Fig. 1. The block diagram of far-field antenna range with the terrain profile between transmitter and point of observation $P$ (test antenna).

\section{Antenna Far-Field Range}

The operation of the antenna should almost always be measurement over some region of solid angle and over some frequency range. The antenna parameters are most often defined by the requirements of a using system and represent areas where operation is critical [2]. The whole far-field measurement range for antenna measurements was completely rebuilt in 2013 including new test equipment and antenna towers. That creates additional constraints, as an owner (producer of radar systems) has to bear in mind the other criteria (such as utilization of existing measurement equipment, non-ideal terrain profile between towers, economy impacts, rebuild deadlines and potential products considering frequency bands). The block diagram of farfield antenna range and the terrain profile between transmitter and antenna under test (AUT), which obviously cannot be changed, is shown in Fig. 1.

Another complication for outdoor ranges is their stray radiated emissions of communications or navigation systems. Furthermore, when the measurements involve proprietary or classified information, achieving a satisfactory level of security may not be possible on an outdoor range. These requirements conflict with the increased radiated signal power necessary to compensate for the $1 / r^{2}$ space attenuation as the AUT should be at distance greater than
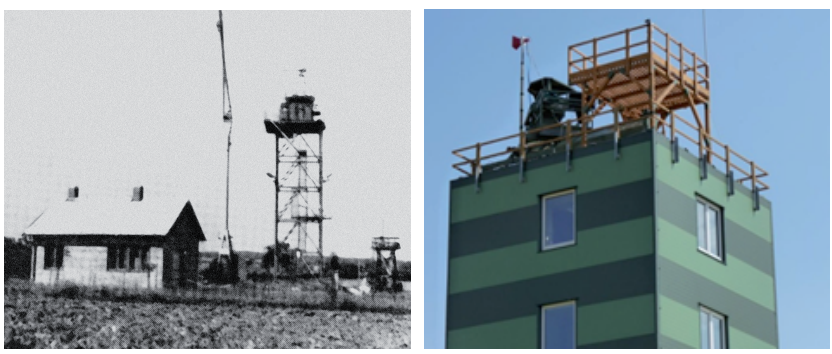

Fig. 2. The initial AUT site of far-field outdoor range (left) and the renewed tower, AUT and vertical positioners. the usual criterion $2 D^{2} / \lambda$, where $D$ is the is the largest dimension of the AUT and $\lambda$ is the wavelength. The initial (1970's) AUT site of far-field outdoor range and the renewed tower, AUT and vertical positioners are shown in Fig. 2. The concrete tower, covered with polyurethane foam and metal sheets, is used to increase mechanical stability.

Optimizing the planarity of the wave-fronts illuminating the AUT at point of observation $\mathrm{P}$ and reducing environmental reflections the new vertical positioner has been designed. That allowed the $5 \mathrm{~m}$ vertical movement of far-field scanning. The relatively large new transmitting antennas for various frequency bands $\left(1-2 \mathrm{GHz}\right.$ with $4^{\circ}$ beamwidth, 2-3 GHz with $2^{\circ}$ beamwidth and $8-10 \mathrm{GHz}$ with $1^{\circ}$ beamwidth) shown in Fig. 3 are used to reduce reflections from the ground and nearby objects. However, that is not ideal due to large AUT. Moreover, the deviation of tower top due to wind and temperature (about $0.1 \mathrm{~m}$ ) is greater than the deviation of concrete tower.

Therefore, completely new measurement campaign has been performed $(2013$ - 2014) to study various conditions such as surface conditions and tilting, which could be used for reduction of reflections from the ground and nearby objects for various frequency bands. Some of preliminary measurements are shown in Fig. 4 - 7.

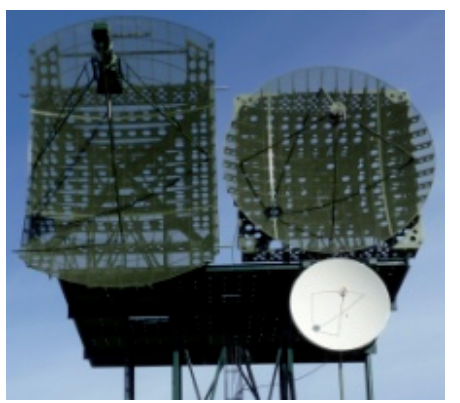

Fig. 3. Transmitting antennas for various frequency bands. 

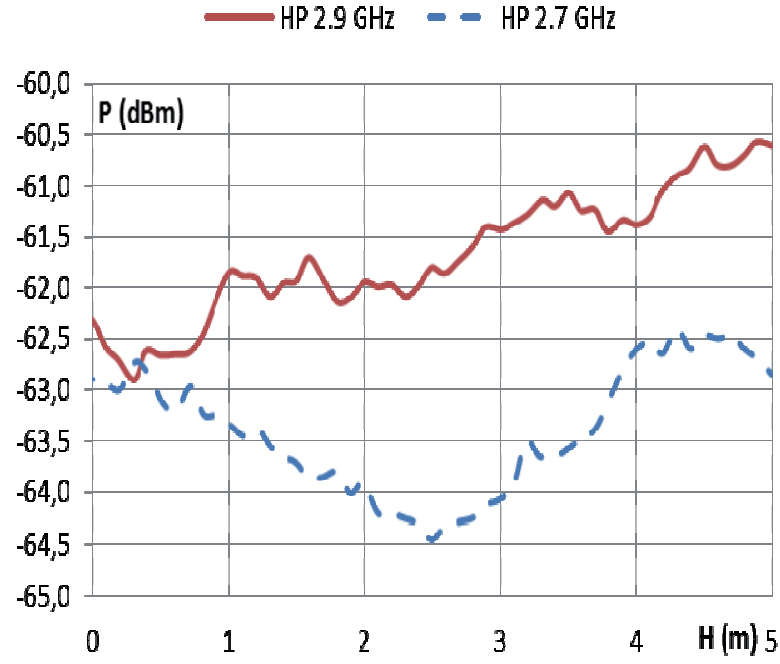

Fig. 4. The measurement of horizontal polarization for 2.9 and $2.7 \mathrm{GHz}$.

$\longrightarrow \mathrm{VP} 2.9 \mathrm{GHz}=-\mathrm{VP} 2.7 \mathrm{GHz}$

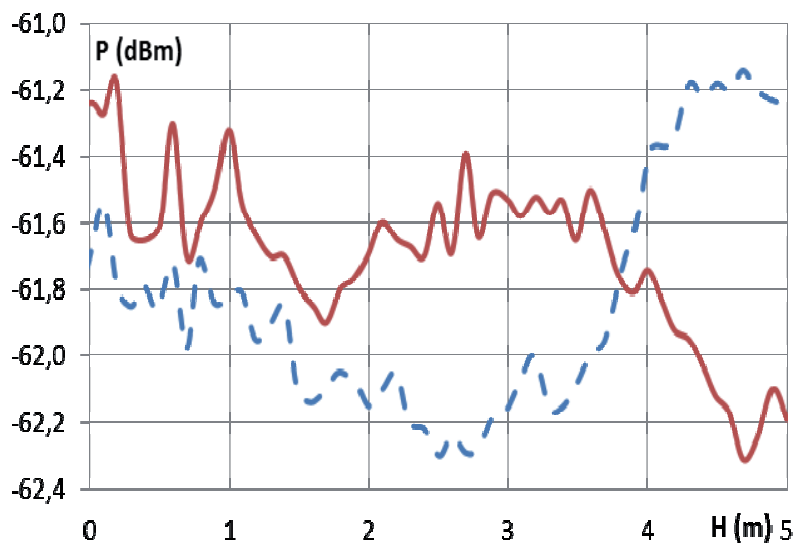

Fig. 5. The measurement of vertical polarization for 2.9 and $2.7 \mathrm{GHz}$.

\section{$=\mathrm{HP} 1.09 \mathrm{GHz}-\mathrm{HP} 1.03 \mathrm{GHz}$}

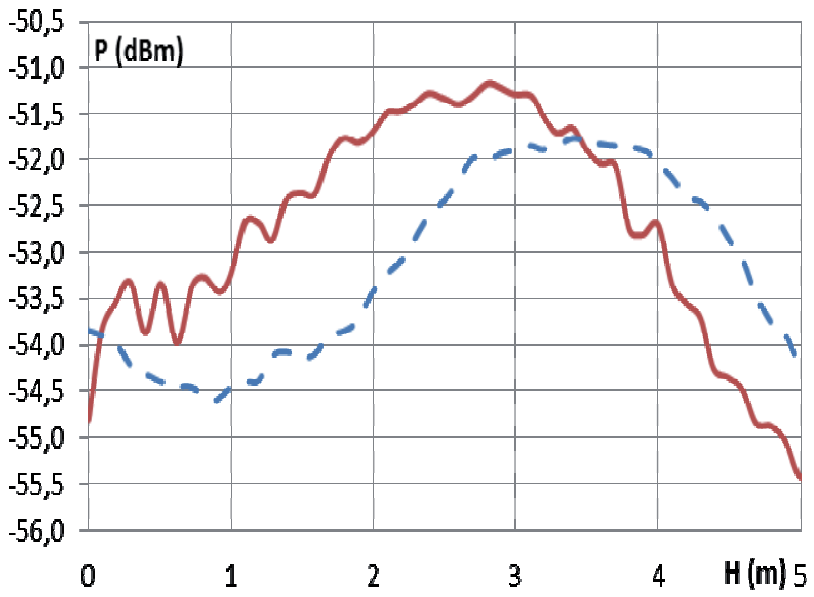

Fig. 6. The measurement of horizontal polarization for 1.09 and $1.03 \mathrm{GHz}$.

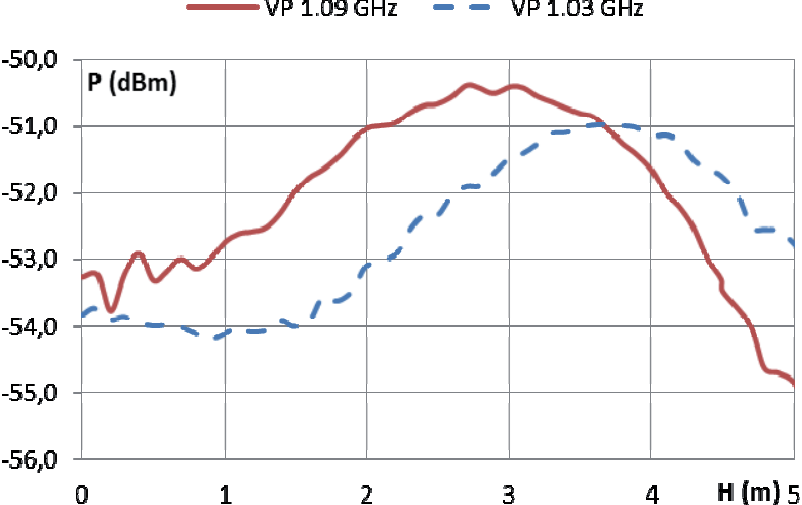

Fig. 7. The measurement of vertical polarization for 1.09 and $1.03 \mathrm{GHz}$.

The measurements were performed during various seasons, and therefore the surface between transmitting and receiving antennas was substantially changing such as relatively dry or wet surfaces. It is obvious that the preliminary performance of far-field range is not satisfactory especially for lower frequency bands as power vertical distributions are not uniform. The improvement could be reached by a change of transmitter antenna tilt as for big transmitting antennas (narrow beam-width) the tilting could reduce environmental reflection. Measurements could be very time consuming, and thus the optimizing of this process could be done by numerical simulations.

\section{Numerical Simulations}

Propagation of electromagnetic waves considering irregular terrain is very challenging for numerous problems such as electromagnetic compatibility, communications, radar coverage, and antenna far-field measuring range. The electromagnetic wave propagation over irregular terrain and optimizing of vertical distribution are briefly described. The geometrical optics and various modifications of geometrical theory of diffraction (GTD) could be used [8] but GTD fails to predict fields at caustics. An integral equation approach [9] is very slow. The algorithms [10] provide considerable savings in processing time for the solution of this equation. The parabolic equation method for describing electromagnetic propagation in vertically stratified troposphere [11] could be used. However, the biggest disadvantage is that very large computer resources are required.

Irregular terrain reflection computations can be found in [12]. The program was tailored for analyses of planned and/or assembled various antenna far-field measurement ranges in the Czech Republic and India. Hoverer thanks to efficient numerical integration, it was possible to solve various problems such as numerical simulations of higher altitude wave propagation, computations of radar coverages and numerous radar site studies (comparison of numerical computations and flight test) [12]-[19]. The gradual improvement of computer resources allows 
extensions and the modifications of initial method. The Franz formula has been used for vector solutions [13]. Physical optics approximation of vector problem [17] offers much more consistent solutions. The scattered field can be divided into two parts namely the reflected radiation component (with the reflection coefficient terms both for smooth and random surfaces) and the shadow radiation component [17], [18]. That allows the calculations of polarization even for the shadow radiation component. The reflected component depends on the local reflection coefficient. On the other hand, the shadow radiation power is equal to the total power incident on a scattering object and it does not depend on the reflection coefficients [20]. The shadow radiation gives origin to edge waves, creeping waves, and surface diffracted rays. The described method actually replaces a real scattering object by the equivalent currents, and therefore it could be much more accurate. The problems of this method are due to approximations accepted for the surface field. That could be diminished using the two-dimensional physical theory of diffraction (PTD), which is a natural extension of physical optics. Moreover, the new version of PTD [21] is valid for all scattering directions especially that could include forward scattering.

Numerical simulations (see [17] for detailed description) have been used for optimizing of the rebuilt antenna range. Fig. 8 shows the comparison of measurements with antenna tilt $1.4^{\circ}$ (HP $2.8 \mathrm{GHz}$ ) and calculations of $A=$ $20 \log \left|E(P) / E_{0}\right|$, where $E(P)$ is the electric field for observation point, $E_{0}$ is the horizontally polarized incident electric vector for frequency of $2.8 \mathrm{GHz}$ and the antenna tilt of $1^{\circ}$ (AT 1), $1.25^{\circ}$ (AT 1.25) and $1.5^{\circ}$ (AT 1.5). Similarly, Fig. 9 shows the comparison of measurements with antenna tilt $2^{\circ}$ (HP $1.03 \mathrm{GHz}$ ) and calculations of $A=$ $20 \log \left|E(P) / E_{0}\right|$ for the horizontally polarized incident electric vector for frequency of $1.03 \mathrm{GHz}$ and the antenna tilt of $1.5^{\circ}$ (AT 1.5 ), $2^{\circ}$ (AT 2) and $3^{\circ}$ (AT 3). Obviously, the best calculated performance is achieved with tilt of $3^{\circ}$. However, the optimization of vertical distribution should be done considering the other conditions. In this case, the vertical dimension of AUT $(0.5<H<1.5 \mathrm{~m})$ along with a nearly maximum of transmitting main beam was the key requirement. Moreover, the deviation of transmitting tower top could create angle changes about $0.5^{\circ}$.

Even if a more detailed description of the parameters of measuring devices, transmitting and receiving antennas and the surrounding environment is a logical requirement that could not be done due to a reasonable paper extent. To demonstrate some properties, the transmitting antenna [22] and some AUT [16], [23]-[25] are described considering both large antennas such as the large vertical aperture (LVA) of phased antenna array of secondary surveillance radar (SSR) with three quite different beams (Sum, Dif and Omega) and dimensions $8.5 \times 1.9 \mathrm{~m}$, and antennas of monopulse conventional SSR with relatively small vertical size. Abundant measured results have been obtained during careful tests of the antenna far-field measurement ranges for various conditions since early 1970's (as for any new antenna type the antenna range vertical distribution was tested with the old analog vertical positioner) and just some comparisons of numerical simulations and experiments were already presented [12]-[17]. The differences could be explained by reflections from objects in the neighborhood of the horn such as a tower structure and guard rails and by reflection coefficient changes, which depends on seasonal terrain conditions.

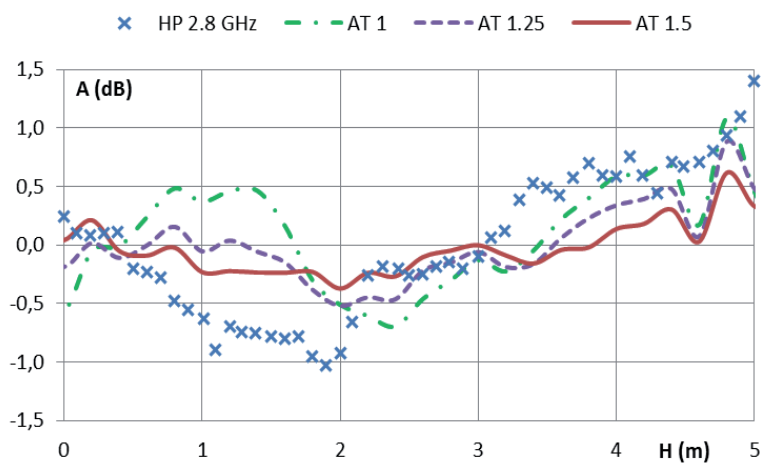

Fig. 8. The comparison of measurements with antenna tilt $1.4^{\circ}$ (HP $2.8 \mathrm{GHz}$ ) and calculations of $A=20 \log \left|E(P) / E_{0}\right|$, where $E(P)$ is the electric field for observation point, $E_{0}$ is the horizontally polarized incident electric vector for frequency of $2.8 \mathrm{GHz}$ and the antenna tilt of $1^{\circ}$ (AT 1$), 1.25^{\circ}$ (AT 1.25 ) and $1.5^{\circ}$ (AT 1.5).

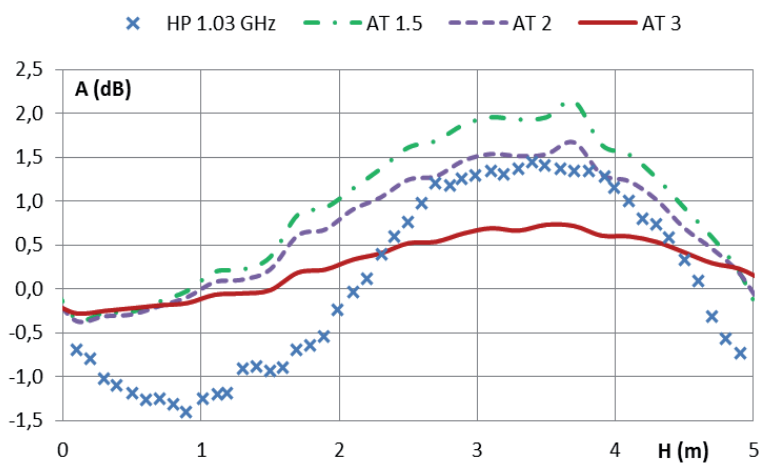

Fig. 9. The comparison of measurements with antenna tilt $2^{\circ}$ (HP $1.03 \mathrm{GHz}$ ) and calculations of $A=$ $20 \log \left|E(P) / E_{0}\right|$, for the horizontally polarized incident electric vector for frequency of $1.03 \mathrm{GHz}$ and the antenna tilt of $1.5^{\circ}$ (AT 1.5), $2^{\circ}$ (AT 2) and $3^{\circ}$ (AT 3).

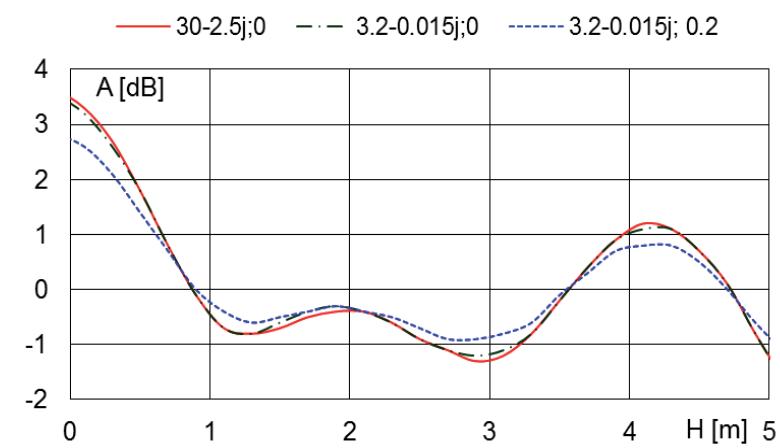

Fig. 10. Numerical simulations for very dry surface $\left(\varepsilon_{0}=\right.$ $3.2-0.015 j)$, very wet surface $\left(\varepsilon_{\mathrm{r}}=30-2.5 j\right)$, and surface with standard deviation $\sigma=0.2 \mathrm{~m}$ and $\sigma=0 \mathrm{~m}$. 
The examples of numerical simulations for various relative permittivity values $\varepsilon_{\mathrm{r}}$ and standard deviations $\sigma$ are shown in Fig. 10.

\section{Conclusion}

This paper describes the design of completely reconstructed antenna far-field range for antenna measurements. That is rather complex task. It is usual that various requirements are quite contradictory. It is not possible to find the best solution of one (even very important) requirement without consideration the other conditions. Therefore, the paper aims are manifold. Firstly, a suitability of a chosen numerical approximation should be demonstrated. Secondly, a possibility of antenna range optimizing is verified by using numerical simulation considering various constraints (some have been briefly mentioned), which cannot be quantitatively considered. The vertical dimension of AUT along with a nearly maximum of transmitting main beam is usually the key requirement, and therefore the numerical values how much the far-field range has been improved via optimization procedure cannot be defined. Thus the complexity of solution is an original result.

The new vertical positioner, shown in Fig. 2, has been designed. That allowed the far-field scanning movement of $5 \mathrm{~m}$. Therefore, the completely new measurement campaign has been performed to study changes of various cases such as changes of frequency, surface circumstances and tilting of antennas. Some of the results are shown in Fig. $4-7$. It is obvious that the "initial" performance of far-field range is not satisfactory especially for lower frequency bands as power vertical distributions are not uniform.

The improvement could be reached by a change of transmitter antenna tilt as for large transmitting antennas with narrow beam-widths the tilting could reduce environmental reflection. However, the narrow beam-widths would be unsuccessful for uniform fields, and therefore transmitting antennas should be selected very carefully. That could be very time consuming, and thus the optimization of this process is done by numerical simulations. The answer is even more complicated as the antenna range should be tailored according to the produced antennas.

The paper briefly reviews the analysis of electromagnetic wave propagation over irregular terrain. The physical optics approximation of vector problem, which allows more reliable computations for low altitude propagations and diffraction field zone without any auxiliary approximate procedures such as knife-edge diffractions, is briefly mentioned. It offers much more consistent solutions, which take into account the polarization. The paper presents the comparison of measured and various calculated values, which are used for the optimization of microwave far-field antenna range. It can be seen that the measurement values are in agreement with the numerical simulations. Numerical simulations clearly offer the advantageous possibilities of antenna tilting shown in Fig. 8 and Fig. 9 especially for lower frequencies. However, the antenna range should be tailored according to the AUT.

The differences could be explained by reflections from objects in the neighborhood of the AUT positioner such as a tower structure and guard rails shown in Fig. 2 and by surface reflection coefficient changes. That depends on terrain conditions (e.g. the earth surface could be covered by snow, plowed or overgrown by vegetation). It affects both reflected and total field but it is not usually substantial as the local reflection coefficient $\Gamma \approx-1$ for a low grazing angle regardless of polarization. However, larger surface roughness could affect the experimental results substantially. That was previously demonstrated by experiments and various numerical simulations.

\section{Acknowledgments}

The research was supported by the "Competence Center of Railway Vehicles" grant No. TE01020038. The authors are thankful to anonymous reviewers, whose suggestions helped a lot in improving the paper.

\section{References}

[1] BALANIS, C. A. Modern Antenna Handbook. 1st ed. Hoboken: John Wiley \& Sons, 2008. ISBN 978-0-470-03634-1.

[2] HOLlis, J. S., LYON, T. J., ClAYTON, L. Microwave Antenna Measurements. 3rd ed. Atlanta, Scientific-Atlanta, Inc., 1985.

[3] YAGHJIAN, A. D. An overview of near-field antenna measurements. IEEE Transactions on Antennas and Propagation. 1986, vol. 34, no. 1, p. 30-45. DOI: 10.1109/TAP.1986.1143727.

[4] BENnETT, J. C., ANDERSON, A. P., MCINNES, P. A., WHITAKER, A. J. T. Microwave holographic metrology of large reflector antennas. IEEE Transactions on Antennas and Propagation, 1976, vol. AP-24, no. 3, p. 295-302. DOI: 10.1109/TAP.1976.1141354.

[5] PUSKELY, J. Application of iterative Fourier method in cylindrical phaseless antenna measurement technique. Radioengineering, Apr. 2012, vol. 21, part 3, no. 1, p. 422-429. DOI: $10.13164 /$ re. 2012 .

[6] RAZAVI, S. F., RAHMAT-SAMII, Y. Resilience to probepositioning errors in planar phaseless near-field measurements. IEEE Transactions on Antennas and Propagation, 2010, vol. AP58, no. 8, p. 2632-2640. DOI: 10.1109/TAP.2010.2050421.

[7] SMITH, D., YURDUSEVEN, O., LIVINGSTONE, B., SCHEJBAL, V. Microwave imaging using indirect holographic techniques. IEEE Antennas and Propagation Magazine, Feb 2014, vol. 56, no. 1, p. 104-117. DOI: 10.1109/MAP.2014.6821762.

[8] LUEBBERS, R. J. Propagation prediction for hilly terrain using GTD wedge diffraction. IEEE Transactions on Antennas and Propagations, Sep 1984, vol. 32, no. 9, p. 951-955. DOI: 10.1109/TAP.1984.1143449. 
[9] HVIID, J. T., ANDERSON, J. B., TOFTGÅRD, J. T., BØJER, J. Terrestrial-based propagation model for rural area-an integral equation approach. IEEE Transaction on Antennas and Propagations. Jan 1995, vol. 43, no. 1, p. 41-46. DOI: 10.1109/8.366349.

[10] AKORLI, F. K., COSTA, E. An efficient solution of an integral equation applicable to simulation of propagation along irregular terrain. IEEE Transaction on Antennas and Propagations. Jul 2001, vol. 49, no. 7, p. 1033-1036. DOI: 10.1109/8.933482.

[11] SKOLNIK, M. I. Radar Handbook. $3^{\text {rd }}$ ed. New York: McGrawHill, 2008. ISBN 978-0-07-148547-0.

[12] SCHEJBAL, V. Computing the electrical field strength of an antenna above an uneven earth. Slaboproudy obzor (in Czech), Dec. 1973, vol. 34, no. 12, p. 541-547. ISSN 0037-668X.

[13] KUPCAK, D. ATC Radar Antennas. Environment Influence on ATC Radar Operation (in Czech), vol. III. MNO, Prague: Czech Rep., 1986.

[14] SCHEJBAL, V. Propagation over irregular terrain. Radioengineering, Apr. 1997, vol. 6, no. 1, p. 19-22. DOI: 10.13164/re.1997.

[15] SCHEJBAL, V. Comparison of propagation over irregular terrain. Radioengineering, Sep. 1997, vol. 6, no. 3, p. 6-9. DOI: 10.13164/re.1997.

[16] SCHEJBAL, V., et al. Czech radar technology. IEEE Transactions on Aerospace and Electronics Systems, Jan. 1994, vol. 30, no. 1, p. 2-17. DOI: 10.1109/7.250400.

[17] SCHEJBAL, V. Improved analysis of propagation over irregular terrain. Radioengineering, vol. 18, no. 1, p. 18-22, Apr 2009. DOI: $10.13164 /$ re.2009

[18] SCHEJBAL, V., FISER, O. Enhanced analysis of propagation over irregular terrain In EuCAP 2010. Barcelona (Spain), 2010, p. 1697-1701. ISBN 978-1-4244-6431-9.

[19] SCHEJBAL, V., GRABNER, M. Investigation of propagation over terrain. In 24th International Conference Radioelektronika 2014. Bratislava (Slovakia), 2014, p. 1-4. DOI: 10.1109/Radioelek.2014.6828454

[20] UFIMTSEV, P. Ya. New insight into the classical Macdonald physical optics approximation. IEEE Antennas and Propagation Magazine, Jun 2008, vol. 50, no. 3, p. 11-20. DOI: 10.1109/MAP.2008.4563560

[21] UFIMTSEV, P. Ya. Improved physical theory of diffraction: removal of the grazing singularity. IEEE Transactions on Antennas and Propagation, Oct 2006, vol. 54, no. 10, p. 2698-2702. DOI: 10.1109/TAP.2006.882179.

[22] ZÁVODNÝ, V., KOPECKÝ, P. Reflector antenna in L band. In Conference on Microwave Techniques (COMITE). Pardubice (Czech Republic), 22-23 April 2015, p. 68-71. DOI: 10.1109/COMITE.2015.7120337.

[23] ZÁVODNÝ, V., KOPECKÝ, P. Measurement of far-field patterns of phased array antennas. In Conference on Microwavwe Techniques (COMITE). Pardubice (Czech Republic), 22-23 April 2015, p. 72-74. DOI: 10.1109/COMITE.2015.7120333.

[24] ZÁVODNÝ, V., KOPECKÝ, P. Omnidirectional antenna cosimulation. In Conference on Microwavwe Techniques (COMITE).
Pardubice (Czech Republic), 22-23 April 2015, p. 72-74. DOI: 10.1109/COMITE.2015.7120337

[25] BEZOUSEK, P., SCHEJBAL, V. Radar technology in the Czech Republic. IEEE Aerospace and Electronic Systems Magazine, 2004, vol. 19, no. 8, p. 27-34. DOI:

10.1109/MAES.2004.1346896.

\section{About the Authors ...}

Ondrej ČERNÝ was born in 1980. He received his Ph.D. degree (2010) in Electric Transport Equipment from the University of Pardubice. Since 2006, he has been with University of Pardubice as a lecturer. He is interested in mathematical simulation, electric drives and control and automation systems. He is the author of papers and publications in this research area.

Radovan DOLEČEK was born in 1971. He received his Ph.D. degree (2006) in Electric Transport Equipment from the University of Pardubice. In 2009, he obtained Doc. degree at the University of Pardubice. Nowadays he works as an associate professor and a head at the Department of Electrical and Electronical Engineering and Signalling in Transport. He is interested in simulations, EMC measurement, power electronics and power system. He is an IEEE member.

Petr KOPECKÝ was born in 1986. He graduated from the Brno University of Technology in 2011. Since 2013 he has been studying doctoral Studies from the University of Pardubice. Since 2011 he has been with the Eldis Pardubice L.T.D. company at microwave design department. $\mathrm{He}$ is interested in radars and antennas measurement.

Vladimir SCHEJBAL graduated from the Czech Technical University, Prague in 1970. He received the PhD degree from the Slovak Academy of Science, Bratislava in 1980. He was with the Radio Research Institute Opocinek, Czech Republic (Antenna Department) from 1969 to 1993. He has been with the University of Pardubice, Czech Republic, since 1994, now as a full professor. He is interested in radars, microwave antennas and propagation. He has published more than 200 papers. He is a senior IEEE member.

Vadim ZÁVODNÝ was born in 1977. He graduated from the Czech Technical University, Prague in 2002. He received the Ph.D. degree from the Czech Technical University, Prague in 2005. He has been with the Eldis Pardubice L.T.D. at microwave design department and since October 2007 to present teaching at University Pardubice, Faculty of Electrical Engineering. $\mathrm{He}$ is interested in radars, microwave antennas and propagation. 